\title{
Status of ATLAS and CMS upgrades on calorimetry and timing
}

Jean-Baptiste Sauvan*, on behalf of the ATLAS and CMS Collaborations

Laboratoire Leprince-Ringuet CNRS/Ecole Polytechnique

E-mail: jean-baptiste.sauvan@cern.ch

The High Luminosity LHC (HL-LHC) will integrate 10 times more luminosity than the LHC, posing significant challenges for radiation tolerance and event pileup on detectors. As part of their HL-LHC upgrade program, the ATLAS and CMS collaborations are replacing the electronics of their calorimeters that provide higher granularity for the level-1 trigger and better measurements of the time of arrival of particles. The CMS collaboration is also designing a High Granularity Calorimeter to replace the existing endcap calorimeters. It features unprecedented transverse and longitudinal segmentation for both electromagnetic and hadronic compartments. This will facilitate particle-flow calorimetry, where the fine structure of showers can be measured and used to enhance pileup rejection and particle identification. In addition, a new dimension in the event reconstruction will be provided by thin timing detectors built between the inner trackers and the calorimeters of both ATLAS and CMS. They will measure the time of arrival of charged tracks with a resolution of 30 to $40 \mathrm{ps}$ and help reducing the pileup density in space-time down to the level of the Phase 1.

Sixth Annual Conference on Large Hadron Collider Physics (LHCP2018)

4-9 June 2018

Bologna, Italy

${ }^{*}$ Speaker. 


\section{Introduction}

The discovery of the Higgs boson at the LHC by the ATLAS and CMS collaborations has opened a new path for the search of physics beyond the standard model. The new scalar boson has been observed in several decay channels [1], in particular in the bosonic channels where its mass and spin-parity properties have been determined. Nevertheless, many pieces of the electroweak symmetry breaking sector still need to be better understood and require much more luminosity than the current phase of the LHC will deliver. This is, for instance, the case for the observation of rare Higgs decays (such as $H \rightarrow \mu \mu$ and $H \rightarrow Z \gamma$ ), to constrain the Higgs boson self-coupling via double-Higgs production, and to perform precise unitarity tests with vector boson scattering. High luminosities at the LHC will in addition extend the searches of new particles at high masses or weak couplings.

The high-luminosity phase of the LHC (HL-LHC) is scheduled to start around 2026 and will accumulate an integrated luminosity of about $3000 \mathrm{fb}^{-1}$. The radiation-induced damages affecting the current calorimeters will require the replacement of their electronics $[2,3]$ or of the full calorimeter in the case of the CMS endcaps $[4,5]$. In addition to being resilient to a very high level of accumulated doses, the new calorimeters will have to face a pileup (PU) of up to 200 simultaneous proton-proton interactions. These requirements have driven the choice of technologies enabling finer granularities as well as precise measurements of the time of arrival of incoming particle showers, in order to disentangle contributions from many interactions. The same need to disentangle multiple interactions justifies also the development of precise timing detectors in both ATLAS and CMS for the time measurement of tracks before they reach the calorimeters $[6,7]$.

The overview of the calorimeters upgrades and timing detectors developments for ATLAS and CMS is presented as well as their performance obtained in simulation and beam tests.

\section{Electronics upgrades of the existing calorimeters}

The ATLAS Liquid Argon (LAr) calorimeter will already be equipped with an upgraded level1 (L1) trigger during the second long shutdown (LS2) of the LHC in 2019-2020. This upgrade provides higher transverse granularity and energy resolution, as well as access to the longitudinal information of the LAr calorimeter. It will replace the current Trigger Tower analog sums (of size $\Delta \eta \times \Delta \phi=0.1 \times 0.1$ ) with the information from each individual layers with a segmentation down to $\Delta \eta \times \Delta \phi=0.025 \times 0.1$ in the front and middle layers. These trigger primitives ("super cells") provide more powerful tools for feature extraction in the L1 trigger. A more effective identification of electrons, photons, and tau lepton showers can be performed thanks to shower shape variables, and the efficiency turn-on curves can also be sharpened using more sophisticated reconstruction algorithms and PU subtraction techniques. The expected improvements in terms of L1 trigger rates and energy resolution for electrons, for an average number of interactions of 80 , are shown in Fig. 1.

In CMS the front-end electronics of the barrel electromagnetic calorimeter (ECAL) will be replaced and will provide a reduction of the shaping time of the signal. This shaping time reduction is a key point to reduce the noise from APD leakage current as well as the contribution from outof-time PU. It also allows for a better discrimination between scintillation signals and "spikes" 

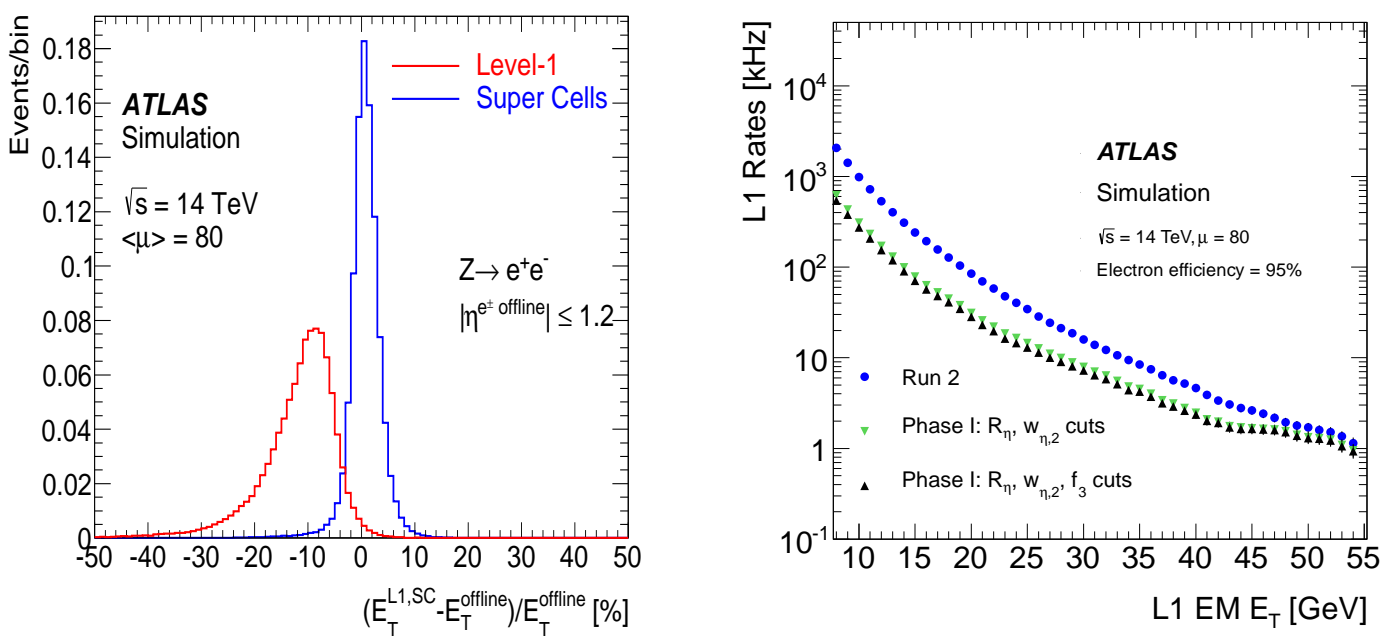

Figure 1: Left: Electron energy resolution with the current ATLAS L1 trigger and with the upgraded Super Cells. Right: L1 electromagnetic (EM) trigger rates as a function of the EM candidate with the current ATLAS L1 trigger and with the upgraded Super Cells with two sets of topological cuts. A full simulation of the ATLAS detector with an average number of $80 \mathrm{PU}$ interactions is used in both cases.

(anomalous signals due to high energy deposits of nuclear fragments in the avalanche photodiodes) and improves the measurement of the arrival time of the signal. The rates of anomalous spike events expected with the upgraded electronics after a pulse shape discriminating variable is applied, as well as the timing resolution as a function of the deposited energy are shown in Fig. 2. In addition, the full crystal granularity will be sent to the off-detector electronics at $40 \mathrm{MHz}$ and used at the L1 trigger, instead of the current Trigger Towers made of the sums of $5 \times 5$ crystals. The back-end electronics will also be upgraded to sustain the higher data rates.
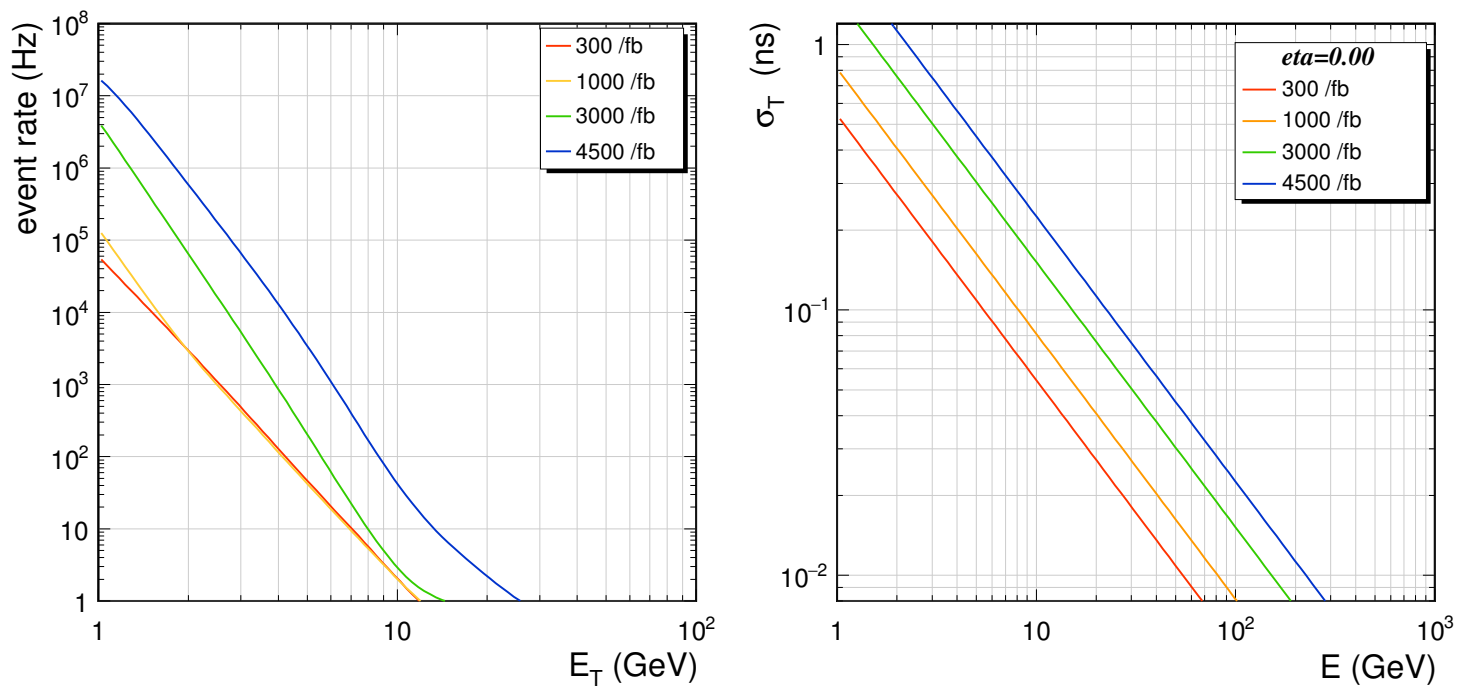

Figure 2: Left: Rate of events containing spike hits above an $E_{\mathrm{T}}$ threshold in the CMS ECAL barrel. Right: Expected time resolution as a function of the deposited energy in the CMS ECAL barrel. Both results are shown after $300,1000,3000$, and $4500 \mathrm{fb}^{-1}$ of accumulated luminosity. 


\section{The High Granularity Calorimeter}

\subsection{Design overview}

The High Granularity Calorimeter (HGCAL) is a sampling calorimeter composed of an electromagnetic (CE-E) and a hadronic (CE-H) section made of 28 and 24 layers of active material respectively. The electromagnetic part uses lead, copper, and copper-tungsten alloy as absorbers while copper and steel are used for the hadronic part. Silicon sensors are used in the region with the highest level of radiation, whilst plastic scintillator with on-tile silicon photomultipliers are used for the part of the CE-H where the radiation level is the lowest. The key parameters of the HGCAL are summarised in Fig. 3.

Surfaces of about $600 \mathrm{~m}^{2}$ of silicon sensors and $500 \mathrm{~m}^{2}$ of scintillator tiles are used. The silicon sensors are finely segmented into 0.5 and $1 \mathrm{~cm}^{2}$ cells with active thicknesses of $300 \mu \mathrm{m}, 200 \mu \mathrm{m}$ or $120 \mu \mathrm{m}$ depending on the region of the detector. These cell parameters are chosen such that the signal-over-noise ratio remains high enough for the measurement of MIP signals (minimum ionizing particles) over the full lifetime of the HGCAL. Scintillator cells of $1^{\circ}$ and $1.25^{\circ}$ are used, arranged in a $\eta-\phi$ grid.

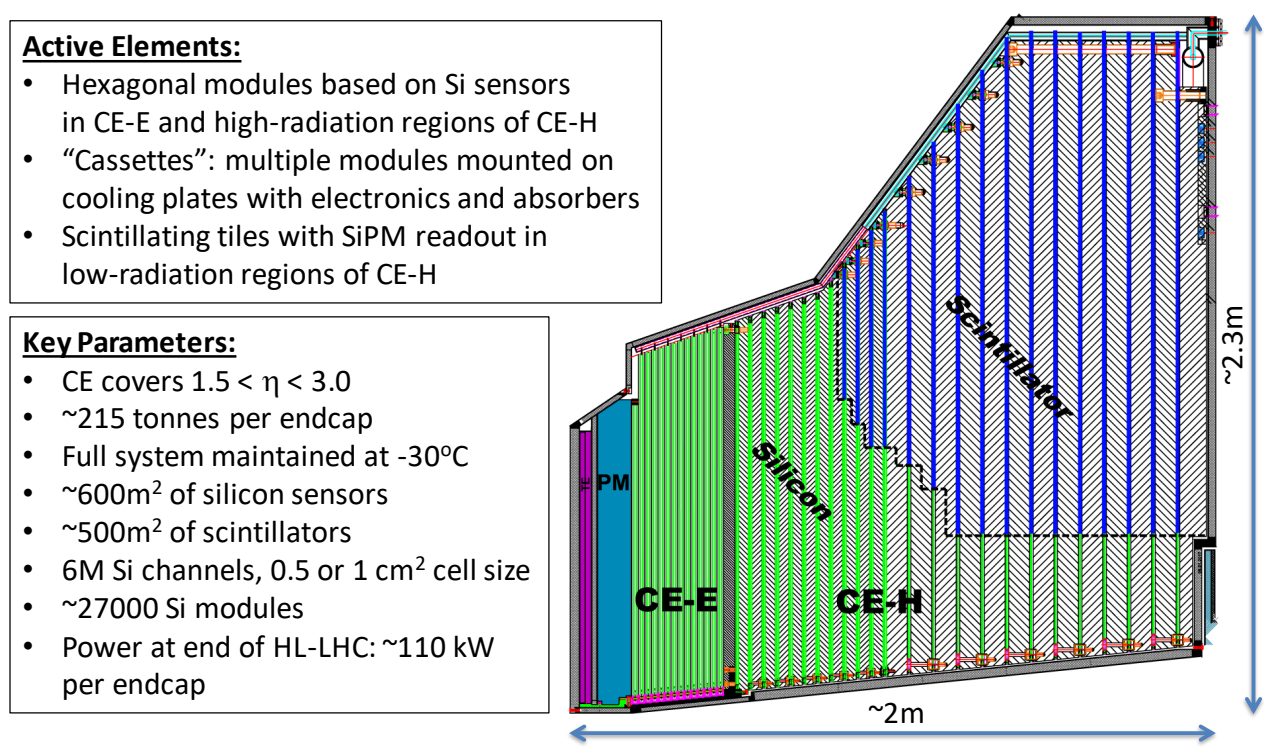

Electromagnetic calorimeter (CE-E): Si, Cu \& CuW \& Pb absorbers, 28 layers, $25 \mathrm{X}_{0} \& \sim 1.3 \lambda$ Hadronic calorimeter (CE-H): Si \& scintillator, steel absorbers, 24 layers, $~ 8.5 \lambda$

Figure 3: Schematic longitudinal cross section of the HGCAL calorimeter and its key parameters. The silicon and scintillator parts of the detector are highlighted in green and dark blue respectively.

For the CE-E part, silicon sensors are sandwiched between copper-tungsten baseplates and PCBs assembled into hexagonal modules, and mounted on both sides of a copper cooling plate. Stainless steel tubes inside the cooling plate carry two-phase $\mathrm{CO}_{2}$ to maintain the silicon sensors at a temperature of $-30^{\circ} \mathrm{C}$. Each cooling plate covers an azimuthal angle of $60^{\circ}$ over the full 
radius of each layer. Plates of lead and stainless steel of the same dimensions cover the sensors and electronics. This assembly is called a "cassette", and 14 cassettes are assembled in a stack to form the CE-E. For the CE-H part, the absorber is made of full disks of stainless steel forming a rigid mechanical structure in which the active elements are inserted as cassettes of $60^{\circ}$. The first 8 layers are fully instrumented with silicon sensors, while the rest of the layers are made of scintillator tiles in the low pseudorapidity region and silicon sensors in the high pseudorapidity region.

For the silicon part, the front-end electronics provides a charge measurement on a large dynamic range, from $0.2 \mathrm{fC}$ to $10 \mathrm{pC}$, as well as a precise timing information with an accuracy better than $100 \mathrm{ps}$ for pulses above $12 \mathrm{fC}$.

\subsection{Reconstruction and physics performance}

The highly granular data provided by the HGCAL, both in the lateral and longitudinal dimensions, as well as the precise timing information, provide detailed inputs to reconstruct efficiently events containing many PU interactions. This fine-grained information can be optimally used by particle-flow algorithms [8] to disentangle the showers from nearby particles and reconstruct their energy and shower properties with a high accuracy.

The energy resolution of electromagnetic showers is an important measure of the detector performance, in particular the value of its constant term, given the high energy of signal particles (e.g., above $100 \mathrm{GeV}$ for typical $\mathrm{H} \rightarrow \gamma \gamma$ photons) in this high pseudorapidity region. Constant terms below $1 \%$ are obtained, assuming an intercalibration of the cells at the level of $3 \%$. The procedure of intercalibration has been carefully studied to ensure that this $3 \%$ precision can be maintained throughout the lifetime of the detector [4].

The transverse size of electromagnetic showers is also an important characteristic of the detector, which has to be low enough to be able to disentangle close-by showers. A Molière radius of about $3 \mathrm{~cm}$ is obtained, with shower sizes below $1.5 \mathrm{~cm}$ in the first layers.

The particle identification is of critical importance in events with a high hadronic activity. Electromagnetic and hadronic showers can be efficiently separated thanks to precise shower-width variables, improved by the capability of reconstructing the principal axes of the showers. The longitudinal dimension can also be used to separate the different development of electromagnetic and hadronic showers. The separation between electromagnetic and hadronic showers using the shower width in the radial direction and a measure of the shower length are shown in Fig. 4.

In addition, the availability of precise timing information will provide a powerful handle to remove contamination from PU showers. The time resolution per cluster below $20 \mathrm{ps}$ for cluster energies above $10 \mathrm{GeV}$ can be used to clean PU contaminations in hadronic jets and to discriminate bremsstrahlung photons from PU neutral pions in the clustering of electron energy deposits.

Although the primary goal of the HGCAL is to reconstruct the energy and position of electromagnetic and hadronic showers, it can also complement muon chambers to tag muons in the forward region, in particular for $|\eta|>2.4$ where all the layers are equipped with silicon sensors. Since it has the sensitivity to measure energies at the level of the MIP, it can detect a muon going through several consecutive layers around extrapolated tracks.

\subsection{Validation in beam tests}

Beam-test campaigns have been conducted with electrons, hadrons and muons of various en- 

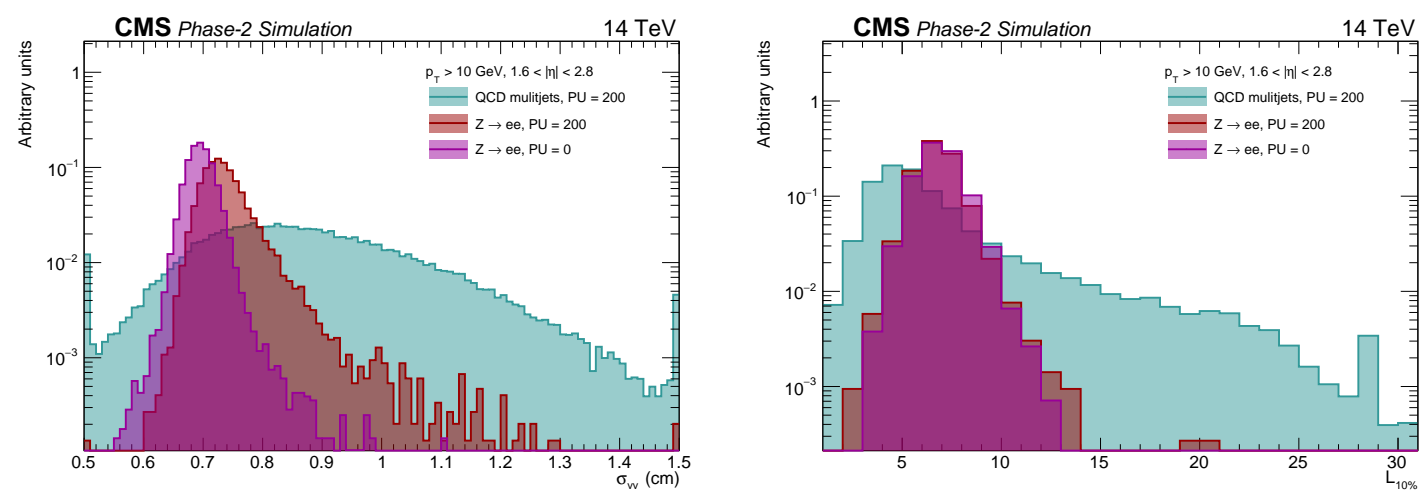

Figure 4: Distributions of the shower transverse width (left) and of the shower depth integrating $10 \%$ of the shower energy (right). The distributions for true electrons from $Z$ decays with $200 \mathrm{PU}$ and without PU (red and purple) and for hadronic jets reconstructed as electrons with 200 PU (blue) are compared.

ergies, to validate the detector concepts and its simulation. Up to 20 modules have been put in beam lines at FNAL and at CERN to probe electromagnetic showers (up to $27 X_{0}$ ) and hadronic showers (up to $4 \lambda$ ). The noise was sufficiently low such that the MIP peak could be extracted with a signal-over-noise ratio of more than 7 (Fig. 5, left) for the intercalibration of all the cells. The reconstructed showers have also been compared with simulated events. The simulation showed a very good agreement both in terms of energy resolution and of shower shapes. As an example the distribution in data and simulation of the ratio of the energies in one ring and two rings of cells $\left(E_{7} / E_{19}\right)$ for hadron showers is shown in Fig. 5 (right).
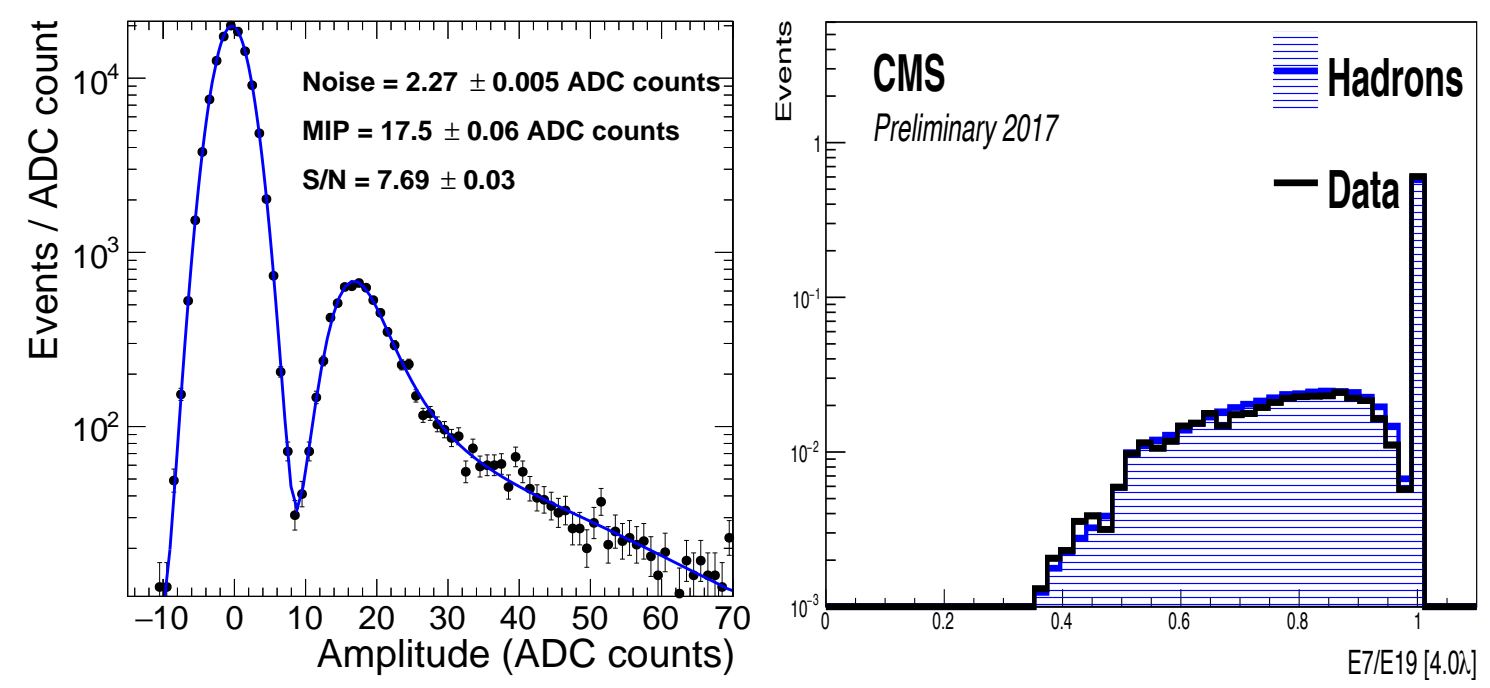

Figure 5: Left: distribution of the amplitude of the pulses in ADC counts for incident charged pions in a typical silicon cell. Right: Distribution of the ratio of energies in one ring $\left(E_{7}\right)$ and two rings $\left(E_{19}\right)$ of cells measured at a depth of $4 \lambda$ for incident charged hadrons. 


\section{Timing detectors}

\subsection{Designs overview}

Both ATLAS and CMS plan to build precise timing detectors in front of their calorimeters in order to measure the time of arrival of charged tracks with a resolution of $30-40 \mathrm{ps}$. While the ATLAS design (HGTD: High Granularity Timing Detector) focuses on the high pseudorapidity region ( $2.4<|\eta|<4$ ), the CMS design (MTD: MIP Timing Detector) will cover the region $|\eta|<3$, including the barrel part. Two different technologies are foreseen for low radiation (barrel) and high radiation (endcaps) regions: silicon-based low gain avalanche diodes (LGAD) will be used in the endcaps of ATLAS and CMS, while LYSO:Ce crystals with silicon photomultipliers will be used in the barrel of CMS.

In order to maintain a $30 \mathrm{ps}$ timing resolution until the end of the HL-LHC data taking, the ATLAS HGTD will be made of 2 disks populated with sensors on both sides of the disks with partial overlaps, effectively yielding an average of 2 hits per track in the region $2.4<|\eta|<3.1$ and 3 hits per track in the most forward region $3.1<|\eta|<4$. The CMS endcap MTD requires only a single disk due to the more central pseudorapidity region covered. Pixels of size $1 \times 3 \mathrm{~mm}^{2}$ in CMS and $1.3 \times 1.3 \mathrm{~mm}^{2}$ in ATLAS are used in the endcaps, for a total of 1.8 and 3.5 million channels respectively.

Detector cells in the barrel of the CMS MTD consists of $11 \times 11 \mathrm{~mm}^{2}$ crystal tiles with varying thickness from 2.4 to $3.75 \mathrm{~mm}$ coupled to $4 \times 4 \mathrm{~mm}^{2} \mathrm{SiPMs}$. The full hermetic coverage is obtained with 250 thousands staggered cells arranged on two layers.

\subsection{Performance}

A timing resolution of the order of $30 \mathrm{ps}$ effectively reduces the pileup density in space-time to the levels of the Phase 1. Timing detectors therefore attenuate largely the impact of increasing pileup on vertex reconstruction, jet energy reconstruction, b-tagging, and lepton isolation. This impact is more pronounced in the endcap region, typically above $|\eta|=2$, as shown in Fig. 6 (left) for the example of the pileup contamination in jets. But although the deterioration of performance with pileup is less pronounced in the barrel region, a timing detector in this region can still have a significant impact, as can be seen in Fig. 6 (right) for b-tagging efficiency.

The new timing capability of the calorimeters will provide a measurement of the time of arrival of particle showers, e.g. electromagnetic showers of photons from the Higgs boson decay. The combination of the time of arrival measured for the two photons can be used to reconstruct their vertex in space-time, even without pointing calorimeters, with a triangulation technique. However this reconstruction breaks down for small rapidity gaps between the two particles. In that case the photon timing information can be combined with the reconstructed vertices obtained from the tracker and timing detectors and the loss of position resolution can be recovered. The impact of the calorimeter timing and of the CMS MTD on the reconstruction of the $H \rightarrow \gamma \gamma$ lineshape is illustrated in Fig. 7 (left).

Timing detectors will also provide a measurement of the velocity of long-lived particles thanks to the reconstruction of primary and secondary vertices in space and time, which can then be converted into peaking mass variables. Long-lived particles appear in many BSM extensions, for 

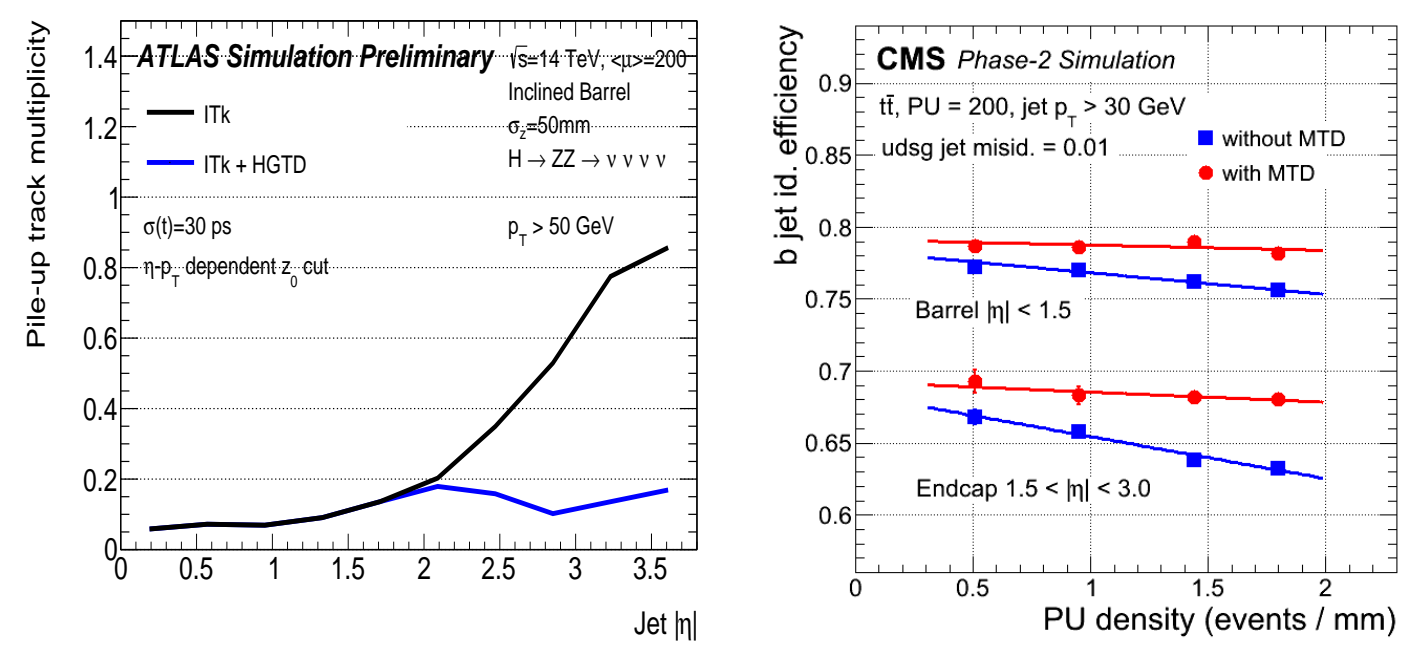

Figure 6: Left: Pileup track multiplicity in reconstructed jets as a function of the pseudorapidity, without and with the ATLAS HGTD. Right: b-jet identification efficiency without and with the CMS MTD, in the barrel and in the endcaps. In both cases a simulation with an average of $200 \mathrm{PU}$ interactions is used.
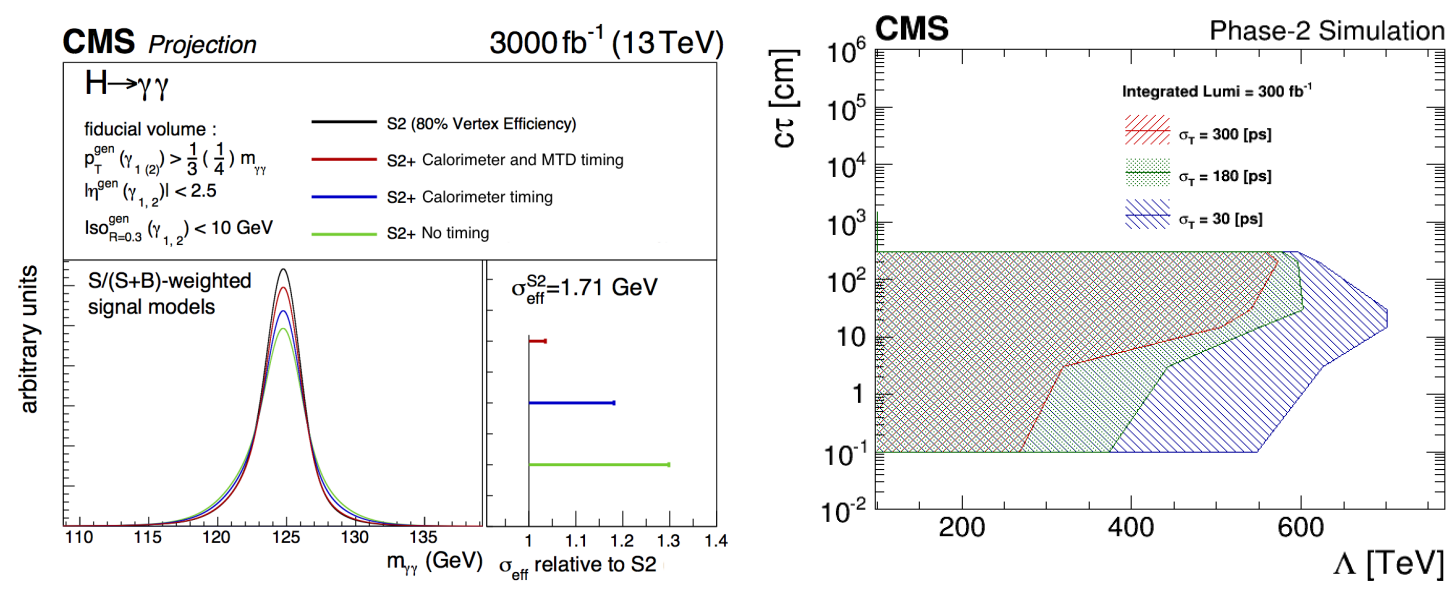

Figure 7: Left: $H \rightarrow \gamma \gamma$ lineshape reconstructed with the Phase-1 CMS detector and with the Phase-2 detector without timing, with the calorimeter timing only, and with both the calorimeter and CMS MTD timing. Right: Sensitivity to gauge-mediated SUSY breaking signals with the Phase-1 CMS detector (300ps resolution), with the Phase-2 detector with calorimeter timing only (180ps), and with the Phase-2 detector including the MTD (30 ps).

instance in gauge-mediated SUSY breaking scenarios, where the lightest neutralino can be longlived and decay to a photon and a gravitino. In that case the photon is produced at some distance from the beam line and its time of arrival can be used to identify signal events. The sensitivity to such signal for different timing capabilities is illustrated in Fig. 7 (right).

\section{Conclusion}

Several parts of the current calorimeters of ATLAS and CMS need to be replaced for the HL- 
LHC, in particular their electronics. New front-end and back-end electronics will provide more granularity for the level-1 triggers and faster signal shaping time. In addition, a highly granular calorimeter is being developed for the replacement of the endcap calorimeters of CMS. Timing detectors will also be inserted between the trackers and the calorimeters to measure the time of arrival of tracks. The technical solutions retained for these detectors are adapted to the extremely harsh radiation and pileup environment. They will provide combined energy, tracking, and timing measurements, which will allow to keep similar performance as during the LHC Phase 1, despite the much higher level of pileup. They will also pave the way to detectors that will run at future colliders.

\section{Acknowledgment}

The work of the author of this paper has been partly funded by the P2IO LabEx (ANR-10LABX-0038) in the framework "Investissements d'Avenir" (ANR-11-IDEX-0003-01) managed by the French National Research Agency (ANR).

\section{References}

[1] ATLAS and CMS Collaborations, Measurements of the Higgs boson production and decay rates and constraints on its couplings from a combined ATLAS and CMS analysis of the LHC pp collision data at $\sqrt{s}=7$ and $8 \mathrm{TeV}$, JHEP 08 (2016) 045

[2] ATLAS Collaboration, ATLAS liquid argon calorimeter Phase-1 upgrade Technical Design Report, CERN-LHCC-2013-017, ATLAS-TDR-022 (2013)

[3] CMS Collaboration, The Phase-2 upgrade of the CMS barrel calorimeters Technical Design Report, CERN-LHCC-2017-011, CMS-TDR-015 (2017)

[4] CMS Collaboration, The Phase-2 upgrade of the CMS endcap calorimeter, CERN-LHCC-2017-023, CMS-TDR-019 (2017)

[5] Edward Scott, Calorimetry at very forward rapidity, This proceeding

[6] CMS Collaboration, Technical Proposal for a MIP timing detector in the CMS experiment Phase-2 upgrade, CERN-LHCC-2017-027, LHCC-P-009 (2017)

[7] Christian Ohm, Fast timing detectors for HL-LHC in ATLAS and CMS, This proceeding

[8] CMS Collaboration, Particle-flow reconstruction and global event description with the CMS detector, JINST 12 (2017), P10003 